\title{
Liberté: Héritage du passé ou idée des Lumières? Edité par Anna Grze Kowiak-Krwawicz et et Izabella Zatorska
}

\section{Regina Bochenek-Franczakowa}

\section{(2) OpenEdition}

1 Journals

\section{Édition électronique}

URL : http://journals.openedition.org/studifrancesi/34727

DOI : $10.4000 /$ studifrancesi.34727

ISSN : 2421-5856

Éditeur

Rosenberg \& Sellier

\section{Édition imprimée}

Date de publication : 1 novembre 2005

Pagination : 419-420

ISSN : 0039-2944

\section{Référence électronique}

Regina Bochenek-Franczakowa, «Liberté: Héritage du passé ou idée des Lumières? Edité par Anna Grze Kowiak-Krwawicz et et Izabella Zatorska », Studi Francesi [En ligne], 146 (XLIX | II) | 2005, mis en ligne le 30 novembre 2015, consulté le 18 avril 2021. URL : http://journals.openedition.org/studifrancesi/ 34727 ; DOI : https://doi.org/10.4000/studifrancesi.34727

Ce document a été généré automatiquement le 18 avril 2021.

\section{cc) $(9)$}

Studi Francesi è distribuita con Licenza Creative Commons Attribuzione - Non commerciale - Non opere derivate 4.0 Internazionale. 


\title{
Liberté: Héritage du passé ou idée des Lumières? Edité par Anna Grze Kowiak-Krwawicz et et Izabella Zatorska
}

\author{
Regina Bochenek-Franczakowa
}

\section{RÉFÉRENCE}

Liberté: Héritage du passé ou idée des Lumières? Edité par Anna GRZE KOWIAK-KRWAWICZ et et Izabella ZATORSKA, Kraków-Warszawa, Collegium Colombinum, 2003. pp. 223.

1 Le volume est le fruit d'un colloque international qui s'est tenu à Varsovie en 2002. L'objectif essentiel des chercheurs a été d'éclaircir les divers aspects d'une notion-clé des Lumières: la liberté, plus particulièrement, la contribution de la pensée européeenne du $18^{\mathrm{e}}$ siècle dans les conceptions de la liberté et l'héritage du passé. La plus grande originalité de ce recueil est de présenter la problématique dans un contexte géographique très large embrassant, à part les grands foyers des Lumières, des pays où les idées nouvelles sont arrivées avec plus ou moins de retard. Ainsi le volume s'ouvre sur un pays lointain, la Chine. Zhang zHILIAN (Three forms of Self-liberation Among Intellectuals in Eighteenth-Century China, pp. 20-25) démontre que si le concept de liberté n'existait pas au $18^{\mathrm{e}}$ siècle en Chine, les intellectuels, sans parler de liberté ou de droits des individus, exprimaient pourtant un certain «esprit rebelle», dans le mode de vivre ou la critique du néo-confucianisme. Marie-Christine SKUNCKE (La Liberté dans la culture politique suédoise au XVIII siècle, pp. 28-41) examine les exemples qui illustrent trois emplois du concept de «liberté» en Suède au $18^{e}$ siècle: la liberté républicaine, soutenue par le parti s'opposant au pouvoir royal absolu; les libertés individuelles des citoyens; le concept monarchiste de «liberté» que le roi Gustave III essaie de récupérer, en rétablissant le pouvoir royal fort. Anna GRZE KOWIAK-KRWAWICZ (Deux libertés, l'ancienne 
et la nouvelle, dans la pensée politique polonnaise du XVIII ${ }^{e}$ siècle, pp. 44-59) présente la spécificité de la problématique en Pologne, où la liberté de participer à la vie publique était de plus en plus identifiée avec la protection des libertés de chaque citoyen; l'auteur passe en revue les propositions des esprits éclairés qui ont tenté des réformes du système politique, dans le cadre des conceptions républicaines. Richard BUTTERWICK (Positive and Negative Liberty in Eighteenth-Century Poland, pp. 62-69) examine une autre conception politique en Pologne, celle de la monarchie limitée, basant son étude sur les conceptions de liberté «positive» et «négative». Martina GRECENKOVA (La liberté et les libertés. Pratiques et concepts de la liberté dans la Bohême des Lumières, pp. 72-85) propose de considérer deux conceptions de liberté et leurs variantes: le «despotisme» du souverain et les libertés des états, le "despotisme» des états et la liberté apportée par l'Etat absolu, dans la réalité du Royaume de Bohême, faisant partie, au $18^{\mathrm{e}}$ siècle, de la monarchie des Habsbourg. Tom VERSCHAFFEL (History and Tradition. Liberty in EighteenthCentury Belgian Historiography, pp. 88-102) explique comment l'élaboration du mythe des Belges, dans l'historiographie du $18^{\mathrm{e}}$ siècle au sud des Pays-Bas d'Autriche a contribué à l'instauration du concept de la nation et de l'état belges en 1830. Raïa ZAïmovA (Histoire et liberté dans les milieux bulgares [fin XVIII ${ }^{e}$-XIXe siècles], pp. 104-113) présente le développement de la conscience de la liberté du pays dans l'historiographie bulgare moderne, depuis l'ouvrage du moine Païssij de 1762 jusqu'à la fin du $19^{e}$ siècle. Dimitris APostolopoulos (De la notion de la liberté pour le monde grec dans la seconde moitié du dixhuitième siècle, pp. 116-120) attire l'attention sur la complexité de la problématique en Grèce: à la fin du $18^{\mathrm{e}}$ siècle, l'exemple exaltant de la liberté «importée» de la France par Napoléon doit y affronter la politique du pouvoir ottoman. Jean MONDT ( «Liberté, liberté(s) chérie(s)", de la liberté héritée à la liberté inventée, pp. 122-134) dégage le thème de la liberté: celle ancienne, héritée, présente dans Egmont de Goethe, et la nouvelle liberté de pensée revendiquée par Schiller dans Don Carlos. Graham GARGET (Jean-Jacques Rousseau, Jacob Vernet and the Enlightened Liberty of Eighteenth-Century Geneva, pp. 136148) caractérise la spécificité des idées politiques dans les écrits des célèbres Genevois, J. J. Rousseau et J. Vernet. Simon DAVIES (Bernardin de Saint-Pierre and the idea of the underprivileged, pp. 150-159) rappelle que l'auteur de Paul et Virginie fut toujours sensible au lien entre la pauvreté des sous-privilégiés de la société et le manque de liberté et de dignité. Renate ZEDINGER (Quelle liberté pour un empereur? François de Lorraine (1708-1765), l'homme privé et son rôle dans la vie officielle de la monarchie habsbourgeoise, pp. 162-174) brosse le portrait d'un personnge historique méconnu, dont l'auteur souligne ici les divers mérites dans la vie politique et économique des pays qu'il avait gouvernés. HansJürgen LÜSEBRINK (La mise en scène théâtrale de la Liberté - la tragédie «Brutus» pendant la Révolution française, pp. 176-192) se penche sur les avatars scéniques et iconographiques du personnage de Brutus dans les divers moments de la Révolution française, révélateurs des idéaux du patriotisme républicain. Erica J. MANUccI (Liberté d'expression et censure sous la Révolution: le cas du théâtre, pp. 194-208) démontre que la censure pendant la Révolution française a posé, pour la première fois, «le dilemme moderne de la liberté: l'instauration de la liberté emporte souvent avec elle la peur de la liberté» (pp. 207-208). Le volume se caractérise par la diversité des approches et points de vue. La problématique de la (des) liberté(s) au $18^{\mathrm{e}}$ siècle, considérée ici par le biais de la confrontation spatiale (le contexte géographique élargi) en même temps que temporelle (le dialogue entre la tradition et l'innovation), acquiert des sens multiples qui méritent notre attention. 